\title{
QoS Routing in Multi-hop Wireless Networks: a New Model and Algorithm
}

\author{
Antonio Capone ${ }^{1}$, Luca Coletti ${ }^{2}$, Marco Zambardi ${ }^{2}$ \\ ${ }^{1}$ Politecnico di Milano \\ piazza L. da Vinci 32, 20133, Milano, Italy \\ capone@elet.polimi.it \\ ${ }^{2}$ Siemens Mobile Communications S.p.A, \\ Via Monfalcone 1, 20092 Cinisello Balsamo - Milan, Italy \\ Luca.Coletti@siemens.com, Marco.Zambardi@icn.siemens.it
}

\begin{abstract}
The definition of new radio network topologies and deployment concepts capable of providing a ubiquitous radio coverage area for beyond $3 \mathrm{G}$ systems is one of the main objectives of the WINNER project [1]. Within this context, routing and forwarding algorithms for infrastructure-based multi-hop networks are investigated. We first present a new mathematical model of the routing problem in infrastructure-based multi-hop wireless networks that takes into account QoS requirements considering bandwidth constraints. Since the characteristics of such new networks allow to control the path selection of each flow, we then propose a new routing algorithm based on a heuristic. Finally, to show the performance of the proposed algorithm several simulation results are presented.
\end{abstract}

\section{Introduction}

Actual 3G wireless technologies seems not able to provide different kind of wireless services and applications to end-user with ubiquitous coverage and complete transparency. For this reason, research community has been started a huge effort in order to develop a new radio access network able to provide wireless access for a wide range of services and applications across all environments with one single adaptive system for all application scenarios and radio environments [1]. A promising new architecture envisioned is the Fixed Relay Network (FRN), where many advantages are expected in terms of coverage, flexibility, throughput and QoS provisioning. A FRN is constituted by fixed transceivers, called relays, that establish a wireless mesh topology connected through Access Points (AP) to a core network. In such network support of multi-hop is mandatory and efficient routing algorithms are required to exploit at best network resources and to efficiently manage internal connections and load from and to the core network.

The problem of routing in multi-hop wireless networks has been deeply investigated in the research area of Mobile Ad-hoc NETworks (MANET). These networks are characterized by a varying topology and require a completely distributed operation of routing protocols. Routing mechanisms for MANETs usually aim at 
minimizing the protocol overhead needed to manage frequent topology changes by means of on-demand route formation [2][3][4] or hierarchical schemes [5]. Therefore, route selection is based on non accurate network state information, and the metrics adopted try to optimize the energy consumption rather then the use of radio resources.

In this hostile network environment the challenge of providing Quality of Service (QoS) guarantees to traffic flows has attracted attention by the research community [6][7] and several algorithms have been proposed [8]-[14]. QoS routing algorithms proposed so far for MANETs are tailored to specific MAC (Medium Access Control) layers able to provide information on resource availability and to control resources assigned to traffic flows. The more common approach is to consider a TDMA (Time Division Multiple Access) based ad-hoc network [13]. Each connection specifies its QoS requirement in terms of time slots needed on its route from source to destination. For each connection, the QoS routing protocol selects a feasible path based on the bandwidth availability of all links and then modifies the slot scheduling of all nodes on the paths. This task is not easy and even the calculation of the residual bandwidth along a path is NP-complete [13] since it can be easily shown that the slot assignment problem is equivalent to the graph coloring problem [15].

Even if FRNs are multi-hop wireless networks like MANETs, their characteristics make the routing problem quite different [16]. FRNs have a defined topology and topology changes are mainly due to node failures that are usually not frequent. Therefore, the distribution of network state information is not much costlier than in wired networks, and even a centralized control of route selection can be adopted [17]. Finally, energy consumption is not a problem for network nodes.

In this paper we propose a new model for the QoS routing problem in multi-hop wireless networks with bandwidth constraints and present an algorithm for its solution suitable for FRNs. The model is an extension of the well known multi-commodity flow problems [18] where link capacity constraints are replaced with new ones that takes into account interference constraints among different radio links. It guarantees that the rates of routed flows are compatible with radio channel capacity, but does not require to explicitly solve the scheduling problem. To solve the proposed problem we present a routing algorithm, Wireless Fixed Relay routing (WiFR), based on an adhoc heuristic. Then, we show how it is possible to derive an admissible scheduling of packet transmissions on the paths provided by the routing algorithm.

The paper is structured as follows. In Section 2 the new mathematical programming model is presented and discussed. Section 3 is devoted to the new routing algorithm, while Section 4 to the scheduling procedure. Section 5 presents numerical results on the performance of the proposed approach. Finally, Section 6 concludes the paper.

\section{Wireless Multi-hop Routing Problem}

The model of the wireless multi-hop routing problem here presented is an extension of the well known multi-commodity flow problem where the network is represented by a graph with a capacity associated with each link and connections are 
represented as flows that compete for the limited link capacities. The objective is to maximize the fraction of offered traffic admitted in the network. A survey on multicommodity flow problems can be found in [18]. Differently from wired networks, with wireless multi-hop networks we cannot associate a capacity to each link in the graph, since parallel transmissions on different links may be prevented due to interference and the inability of stations to transmit and receive at the same time. We assume that when a transmission occurs on a link between a couple of nodes, the transmissions of nodes directly connected to the transmitting or the receiving nodes are prevented. Such an assumption assure that the hidden terminal problem does not occur both for the considered transmission and that of the acknowledgement packet, like for IEEE 802.11 systems.

Let us consider a graph $G=(V, E)$ whose $\mathrm{N}$ vertexes, $\mathrm{V}=\{1,2, \ldots, \mathrm{N}\}$, represent the wireless stations and the $\mathrm{M}$ edges $(i, j)$ connect stations within transmission range.

Consider now a set of $K$ pairs of vertexes $\left(s^{k}, t^{k}\right)$ for $\mathrm{k}=1,2 \ldots \mathrm{K}$ representing the source and destination node associated with $K$ commodities for which a path should be found on the graph $G$. For each commodities $K$ can be introduced the following variables:

$$
\begin{aligned}
& f_{i, j}^{k}=\text { Units of flow of commodity k routed on link from } \mathrm{i} \text { to } \mathrm{j} ; \\
& F^{k}=\text { Total units of flow to be sent from } s^{k} \text { to } t^{k} ; \\
& f_{i, j}=\sum_{k=1}^{K} f_{i, j}^{k}=\text { Total units of flow on arc from } \mathrm{i} \text { to } \mathrm{j} .
\end{aligned}
$$

Then for each node $n$, represented in $G$ by a vertex $n$, the following sets are defined:

$$
\begin{aligned}
& A(n)=\{j \in V \mid(n, j) \in E\} \\
& B(n)=\{j \in V \mid(j, n) \in E\}
\end{aligned}
$$

where $A(n)$ represents the set of nodes that can be reached from node $n$ while $B(n)$ is the set of nodes that can reach node $n$ with their transmission.

As mentioned before, the objective in solving routing problem is to maximize the fraction of total commodities that is routed through the network from source to destination. This can be expressed introducing a parameter $\alpha$ that represents for all commodities the fraction of $F^{k}$ admitted in the network. Therefore objective is to find the optimum $\alpha$, denoted as $\alpha^{*}$, such that for each one of the given $K$ commodities it is possible to route in the network exactly $\alpha^{*} \cdot F^{k}$ units of flow from the source node $s^{k}$ to destination $t^{k}$. The optimum $\alpha^{*}$ is that particular value of $\alpha$ that satisfy the following objective function:

$$
\operatorname{Max}\left\{\sum_{k=1}^{K} \alpha \cdot F^{k}\right\}
$$


Further, conservation equations and non negativity must be satisfied by all flows:

$$
\begin{gathered}
\sum_{j \in A(n)} f_{n, j}^{k}-\sum_{j \in B(n)} f_{j, n}^{k}=\left\{\begin{array}{c}
\alpha \cdot F^{k} \text { if } S^{k}=n \\
-\alpha \cdot F^{k} \text { if } t^{k}=n \\
0 \text { otherwise }
\end{array}\right. \\
f_{i, j}^{k} \geq 0 \text { for all }(i, j) \in E \\
\alpha \in[0,1]
\end{gathered}
$$

The equation (4) states for each commodity $k$ that the difference between incoming flow and outgoing flow on a given node $n$ is positive if node $n$ is the source of the commodity, is negative if node $\mathrm{n}$ is the destination and is equal to zero if node $n$ is an intermediate node of path $k$. Equation (5) states that commodities must be nonnegative.

To model the capacity and interference constraints, new sets of nodes are introduced: the set $S_{i}{ }^{l}$ is made up by all links that are adjacent to node $i$ :

$$
S_{i}^{1}=\{(i, j), j \in V\}
$$

and for each node $j \in A(i)$, the set $S_{i, j}^{2}$ including all links that have one of their end in $A(i)$ and meanwhile do not belong to $S_{i}^{1}$ :

$$
S_{i, j}^{2}=\{(j, k) \mid k \neq i\}
$$

Obviously for the given node $i$, exactly $|A(i)|$ set of this type exist.

Starting from these sets, it is possible to build up the set $G_{i}$ formed by all relays within two hops from relay $i$, this set can be expressed as:

$$
G_{i}=\left\{S_{i}^{1} \cup \bigcup_{j \in A(i)} S_{i, j}^{2}\right\}
$$

It is possible to characterized each set $G_{i}$ by a theoretical capacity $C_{i}$ that represents the maximum aggregate flow that can be routed over the whole set of links belonging to that group. All flows routed on links belonging to set $S_{i}{ }^{1}$ contributes in consuming common resources associated to the whole group $G_{i}$ and to bound possibilities for new transmissions/receptions of relay i. About the various set $S_{i, j}^{2}$ build on each neighbor of node $i$, only the heaviest loaded one should be considered for writing the 
new QoS constraint, since it is the one that gives the more restrictive condition about $G_{i}$ 's resources consumption and about limitation for further transmissions/receptions of node i. It is hence possible to write the constraint to be inserted in the mathematical model as follow:

$$
\sum_{k=1}^{K} \sum_{(i, j) \in S_{i}^{1}} f_{i, j}^{k}+\max _{j \in A}\left(n_{i}\right)\left\{\sum_{k=1}^{K} \sum_{(j, l) \in S_{i, j}^{2}} f_{j, l}^{k}\right\} \leq C_{i} \forall G_{i}
$$

It is possible to transpose the non linear constraint (10) to a set of linear constraints without any approximation obtaining in this way a linear model for our problem. This is done eliminating the max operator that gives the non linearity and extending the constraint to all set $S_{i, j}^{2}$ related to relay $i$ comprising in this way also the heaviest loaded one that gives the more restrictive condition. This set can be compacted in the following expression:

$$
\sum_{k=1}^{K} \sum_{(i, j) \in S_{i}^{\mathrm{l}}} f_{i, j}^{k}+\sum_{k=1}^{K} \sum_{(j, l) \in S_{i, j}^{2}} f_{j, l}^{k} \leq C_{i} \forall S_{i, j}^{2} \in G_{i}, \forall G_{i}
$$

It is now possible to write the mathematical model used to describe problem of QoS routing in a network composed by fixed/movable relays equipped with omnidirectional antennas and a unique radio interface:

$$
\operatorname{Max}\left\{\sum_{k=1}^{K} \alpha \cdot F^{k}\right\}
$$

so that

$$
\begin{gathered}
\sum_{j \in A(n)} f_{n, j}^{k}-\sum_{j \in B(n)} f_{j, n}^{k}=\left\{\begin{array}{c}
\alpha \cdot F^{k} \text { if } s^{k}=n \\
-\alpha \cdot F^{k} \text { if } t^{k}=n \\
0 \text { otherwise }
\end{array}\right. \\
f_{i, j}^{k} \geq 0 \text { for all }(i, j) \in E \\
\alpha \in[0,1] \\
\sum_{k=1}^{K} \sum_{(i, j) \in S_{i}^{1}} f_{i, j}^{k}+\sum_{k=1}^{K} \sum_{(j, l) \in S_{i, j}^{2}} f_{j, l}^{k} \leq C_{i} \forall S_{i, j}^{2} \in G_{i}, \forall G_{i}
\end{gathered}
$$


This new approach results in the complete separation between construction of route that satisfy the required QoS and the definition of proper scheduling at MAC level. This means it could be even applied to MAC that does not support strict resource control, like e.g. IEEE 802.11, taking a proper margin on the radio link capacity in order to take into accounts all overheads due to the protocol and the contention on the channel. In this case if strict control on the flows entering the network is enforced, the new approach assures that no persistent congestion occur in the network.

\section{WiFR: a new QoS routing algorithm for FRN}

To solve the problem presented in previous section we propose a new QoS routing algorithm named Wireless Fixed Relay routing (WiFR). We present WiFR assuming that route computation can performed at a central controller, even if using a proper routing protocol to distribute link usage information, the algorithm could be executed in a distributed way. Since path selection is performed flow-by-flow, the algorithm can be used for on-line routing of connection requests.

The following information is considered and maintained by the routing controller:

- Topology matrix $\boldsymbol{T}$ that is a $N \times N$ matrix where $N$ is the number of relays and the generic element $t_{i k}$ is equal to 1 if $j$ and $k$ are connected and 0 otherwise.

- The set neighbors of any relay $j, H_{l}(j)$, and the set of relays that are two hops away, $\mathrm{H}_{2}(j)$.

- The residual capacity matrix $\boldsymbol{R C}$ that is a $N \times N$ matrix. The generic element $r c_{j, \mathrm{k}} \in[0,1]$ of the matrix represents the fraction of residual capacity on radio link $(j, k)$. The bandwidth $B$ provided by lower layers is an input parameter at routing level.

- Weight matrix $\boldsymbol{W}$ that is a $N \times N$ matrix representing a set of weights associated with network links. These weights are dynamically updated by the algorithms and used for route computation. Weights are defined as:

$$
W_{j, k}=\frac{1}{r c_{i k}^{2}}
$$

if $(i, k)$ is an edge of the network graph, and 0 otherwise.

- Status tab $\underline{s}$ that is a list of record that has $N$ entries used to stored information on relay states. For a given relay $j$ the parameters stored in its record are: $u b(j)$, the fraction of bandwidth consumed either for relay $\mathrm{j}$ transmissions or for receptions of other signals addressed to $j$ or not, $f r(j)$, the fraction of bandwidth that relay $j$ has still available to receive without conflicts with its own transmissions or with other received signals in the set $H_{1}(j), f t(j)$, the fraction bandwidth that relay $j$ has still available to transmit without conflicts with transmissions of other relays in sets $H_{1}(j)$ and $H_{2}(j)$.

Suppose now that a route request from relay $s$ to relay $t$ with a request bandwidth $\beta$ (normalized to the provided bandwidth $B$ ) is delivered to the central entity. The central entity applies the route searching routine that selects, just among the feasible 
paths between source node and destination one, the path that has the minor impact on network global saturation level. We use as basis for our route searching routine the mechanism of Dijkstra algorithm, where the metric is based on weights $w_{j k}$. However, route selection is performed only among feasible paths in a greedy manner. This can be done with the following exploring routine that we developed to determinate if, starting form generic relay $j$, a potential next hop relay $k$ should be explored by Dijkstra algorithm or not. The control steps executed by our routine are:

- If $j$ is the source node and $f t(j)<\beta$ then connection is refused. In this case source $j$ has not enough transmission resources.

- If $j$ is the source node, $k$ is not the destination node, and $u b(j)+2 \beta>1$ connection is refused. In this case source $j$ has not enough bandwidth to transmit to $k$ and then receive $k$ transmission to next node without conflicts.

- If node $k$ is the destination node it can be selected.

- If $f t(k)<\beta$ then $k$ is discarded. In this case $k$ has not enough bandwidth to forward the flow.

- If $i$ is a common neighbor of $j$ and $k$ and $f r(i)<2 \beta$ then $k$ is discarded. In this case node $i$ cannot receive without conflicts the transmissions of $j$ and $k$.

- If destination node $t$ is a neighbor of $k$ and $u b(k)+2 \beta<1$ then node $k$ is selected, otherwise it is discarded.

- If destination node $t$ is not a neighbor of node $k$ and $u b(k)+3 \beta<1$ then node $k$ is selected, otherwise it is discarded.

If the routine states that node $k$ can support the new flow then it is explored by Dijkstra algorithm using the metric defined by weights $w_{j k}$. If the routine instead states that relay $k$ is not able to support the new flow no further actions are taken on relay $k$. In both cases, after having executed proper actions basing on routines response, a new neighbor of the actual selected node $j$ is chosen, if exist, and routine is run again. Once all neighbors of $j$ have been considered, according to Dijkstra algorithm, a new node $j^{*}$ is selected to be included in the shortest path tree. If this node is the destination $t$, then the algorithm terminates, otherwise it is restarted considering the node $j^{*}$. Algorithm steps are repeated until destination is reached or until no node can be added. If a feasible path $\boldsymbol{p}$ is found, central controller updates the bandwidth usage information:

$$
\begin{gathered}
u b(j)=u b(j)+\beta \quad \forall j \mid j \in p, j \neq t \\
u b(k)=u b(k)+\beta \quad \forall k \mid k \in H_{1}(j), j \in p, j \neq t \\
f r(j)=1-u b(j) \quad \forall j \in V \\
f t(j)=1-\max \left\{\max _{k \in H_{1}(j)} u b(k), u b(j)\right\}
\end{gathered}
$$

Equation (18) represents resources consumption of relay forming the new path for transmitting the new flow. Equation (19) represents resource consumption due to the 
physical broadcast: all neighbors of transmitting relay receive the signal. Equation (20) updates the residual capacity for receptions of each relay. Equation (21) updates the residual capacity for transmission of each relay. Also residual capacity values are updated:

$$
r c_{j k}=\min \{f t(j), f r(k)\} \quad \forall(j, k) \in E
$$

and weight matrix $\boldsymbol{W}$ is updated accordingly.

This basic heuristic can be executed online when a new connection request arrives without re-routing already routed flows. In the case the routing algorithm is performed offline on a set of flows, we have developed an optimization routine that tries to reroute the other flows when no feasible path is found for the considered one.

\section{Optimized Scheduling}

We have presented a new mathematical model which route bandwidth guaranteed flows without defining at the same time a scheduling scheme for each node in the network. This allows to apply the new routing approach even to networks based on random access schemes at the MAC layer, like IEEE 802.11, provided that the radio link bandwidth $B$ considered by the routing scheme is properly reduced to take into account all overheads due to collisions and protocol headers. Moreover, if an optimized scheduling scheme can be adopted for a TDMA based MAC layer, it can be obtained in a second phase based on the transmission requirements of each node as defined by the solution provided by the routing procedure. To derive numerical results we have considered and solved this last problem.

Since the path selection has been already performed by the routing algorithm, here the problem is that of assigning slots to network nodes according to the total bandwidth required by the node for originated or relayed flows.

We model this slot assignment problem as a variation of the known Minimum Order Frequency Assignment Problem (MO-FAP) which has been proposed for frequency planning in cellular networks [19].

Let $m(j)$ be the number of slots required for relay $j$. The assumption needed to calculate the number of slots requited by each node is that the bandwidth of flows is a multiple of the minimum bandwidth corresponding to a single slot per frame.

The number of slots in the frame is denoted by $N_{s}$. Binary variables $\mathrm{y}_{\mathrm{c}}$ defines the slots assigned with $\mathrm{y}_{\mathrm{c}}=1$ if slot $c$ is assigned to at least one relay and $\mathrm{y}_{\mathrm{c}}=1$ otherwise, $c=1,2, \ldots, N_{s}$. The objective function is that of minimizing the number of slots in the frame:

$$
\min \left\{\sum_{c \in N_{s}} y_{c}\right\}
$$

Binary decision variables $x_{j c}$ define if slot $c$ is assigned to relay $j\left(x_{j c}=1\right)$ or not $\left(x_{j c}=0\right)$. Obviously, we require that: 


$$
\begin{gathered}
x_{j c} \leq y_{c} \\
\sum_{c=1}^{N_{s}} x_{j c}=m(j)
\end{gathered}
$$

for all $j \in V$ and $1 \leq c \leq N_{s}$. Constraints (24) state that a slot $c$ can be assigned to a relay $j$ only if the corresponding variable $y_{c}$ is set to 1 , and constrains (25) that the number of colors assigned to each relay must be equal to the number of slot required.

We need now to introduce the fundamental constraints that forbid assignment of the same slots to conflicting relay. To define if relay $j$ and $k$ cannot use the same slots, let us define the conflict graph, i.e. a graph $G_{c}\left(V, E_{2}\right)$ where an edge between relay $j$ and $k$ exists if and only if they are prevented to use the same slots. The graph $G_{c}$ for the interference model adopted in previous section can be easily generated. The compatibility constraints are defined as:

$$
x_{j c}+x_{k c} \leq 1 \quad \forall(j, k) \in E_{2}, 1 \leq c \leq N_{s}
$$

Constraints (26) state that if a link between $j$ and $k$ exists in the conflict graph a slot $c$ cannot be assigned to $\mathrm{j}$ and $\mathrm{k}$ at the same time $\left(x_{j c}=1\right.$ and $\left.\mathrm{x}_{k c}=1\right)$.

The slot assignment problem is solved through a heuristic algorithm [19]. The algorithm aims at minimizing the overall number of slot (colors) used so to keep the MAC frame short and the end to end delay low. It is based on a greedy procedure that sorts relays according to their connectivity degree in the graph $G_{c}$. For the sake of brevity the details of the scheduling algorithm are omitted.

\section{Simulation results}

To evaluate the new model and algorithm presented in this paper, the WiFR algorithm, a benchmark QoS routing algorithm based on pure hop-count metric named WSPF (Wireless Shortest Path First) and the optimized TDMA MAC layer as been added into the event-driven network simulator NS-2 [20]. Simulation results presented here has been obtained using two ray channel model, a bandwidth of 2 Mbit/sec, packets of 1 kbyte, exponential On/Off traffic sources with differentiated bandwidth request resembling voice traffic.

Figure 1 shows the throughput obtained in a FRN of 60 relays random deployed following a uniform distribution over a $1000 \times 1000$ meters area for various values of relays radio range. For high values of radio range the network become fully connected and both algorithm gives the same throughput. With networks with low/medium connectivity degree, our routing algorithm not only outperform the WSPF of about $13 \%$, but also rises network capacity of about $19 \%$ with respect to fully connected situation. As the radio range increases, the throughout firstly is reduced till a 
minimum because every single transmission impact on a set $\mathbf{G}_{\mathrm{i}}$ greater and greater rising resources consumption. However, increasing radio range diminishes the route length. For this reason after the minimum the throughput rises till settling to the value corresponding to the fully connected network.

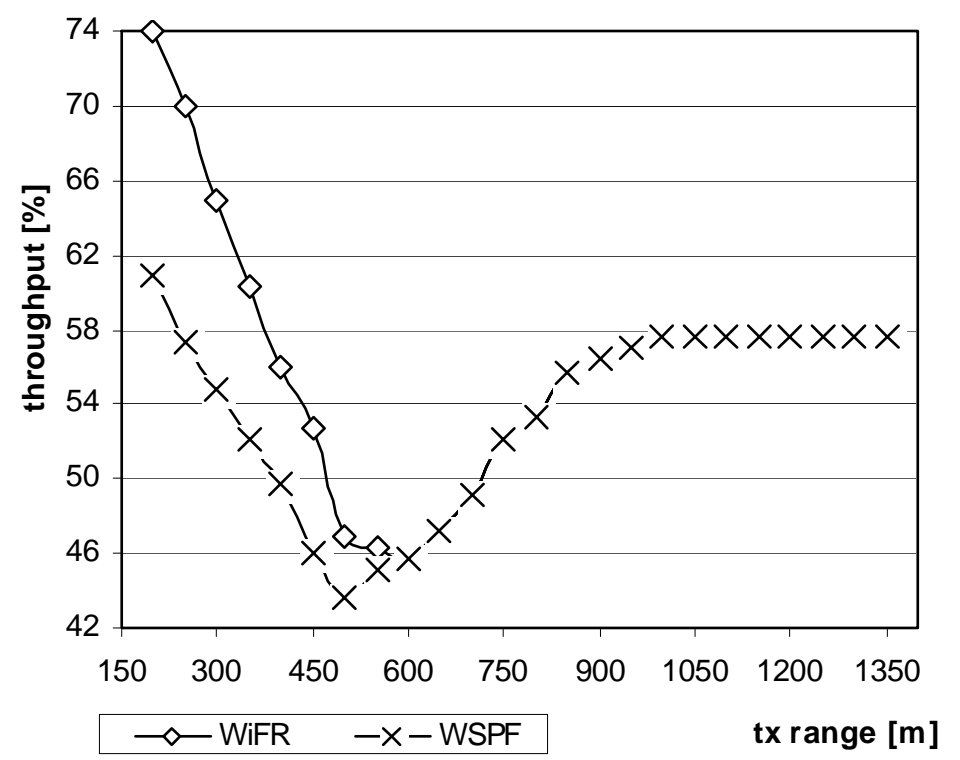

Fig. 1. WiFR vs WSPF, random topologies, 60 relays, high traffic

WiFR performance has been deeply investigated also in Manhattan topologies, see Figure 2, starting from a basic grid of $4 \times 4$ relays used as sources and/or destinations of the connections and adding a number $n$, from $n=0$ (basic grid) to $n=5$, of additional relays that has only the task of forwarding packets.
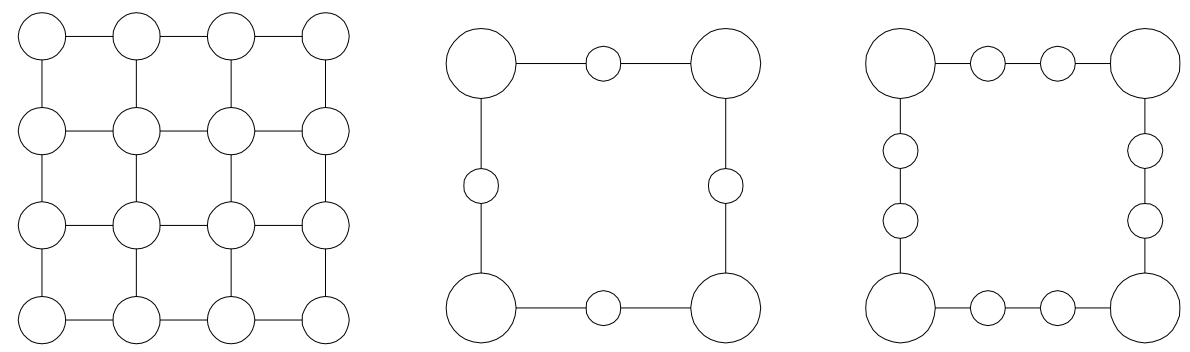

Fig. 2. Manhattan basic grid $(n=0)$ and particular for $n=2$ and $n=5$ topologies

Figure 3 shows that WiFR outperforms WSPF in all Manhattan topologies from $6 \%$ to $10 \%$; further, impact of additional allows to increase throughput thanks to possibility of increasing spatial reuse of shared radio resource, with only one additional relay capacity gain is about $16 \%$, with two additional relays gain is about $20 \%$. It can be noticed that with three and four additional relays throughput decreases 
since route becomes longer and re-transmissions negative effect are not completely balanced by higher spatial reuse. It seems hence that one or two additional relays is the optimal compromise between performance and costs.

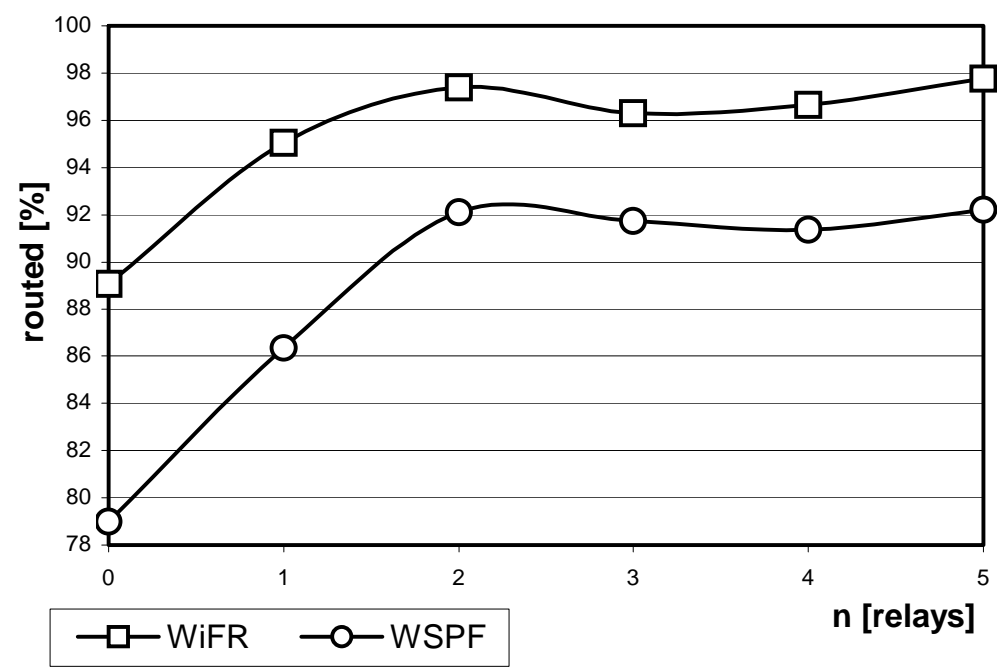

Fig. 3. WiFR vs WSPF, Manhattan topologies, low traffic

Figure 4 shows end-to-end delay achieved when connection requests follow a Poisson process with different inter-arrival time. It can be seen that as the frequency or connection request arrival increase, the end-to-end delay remains stable with slight variation of about $50 \mathrm{msec}$ on an average delay of $0,1-0,2 \mathrm{sec}$. This proofs that WiFR is really able to offer QoS maintaining stable the network condition independently from external offered load.

Finally Figure 5 shows a comparison between WiFR and ad-hoc routing protocols implemented in Ns2, i.e. DSR, DSDV and AODV. Since the ad-hoc routing algorithms do not offer QoS guarantees, simulations has been done with a low traffic level using IEEE 802.11 as MAC layer.

WiFR outperforms all ad-hoc routing protocols of at least $10 \%$ with a maximum of $55 \%$ with respect to DSDV and Manhattan topology with five additional relays. This enforces our idea that FRN shouldn't be considered as a particular case of ad-hoc network, but new routing algorithms should be developed considering the new peculiarity of such scenario. 


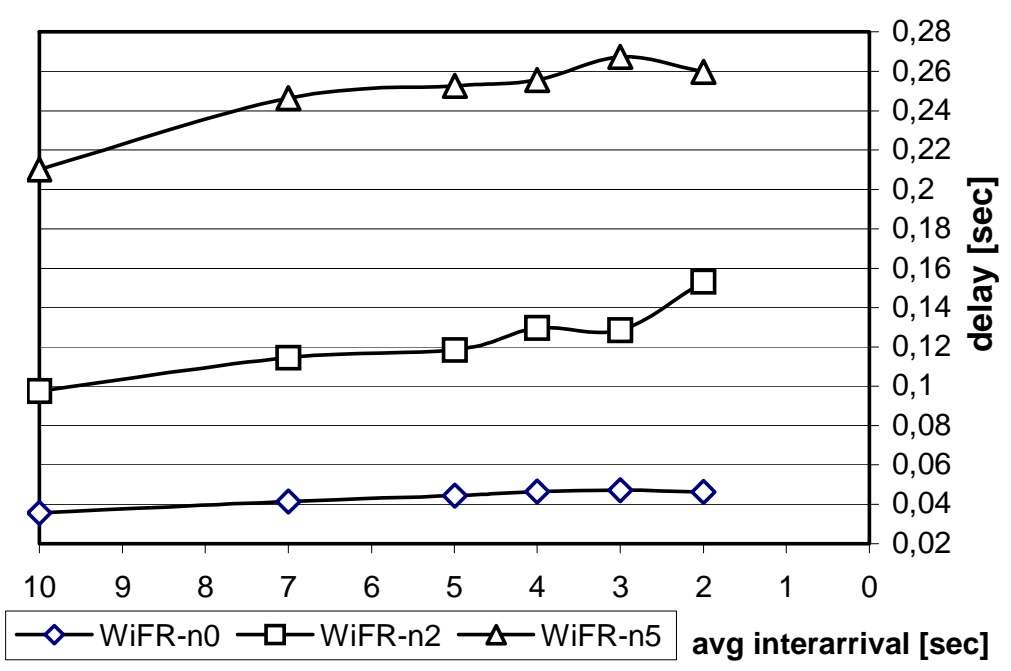

Fig. 4. WiFR, end to end delay with Poisson arrival of connection request

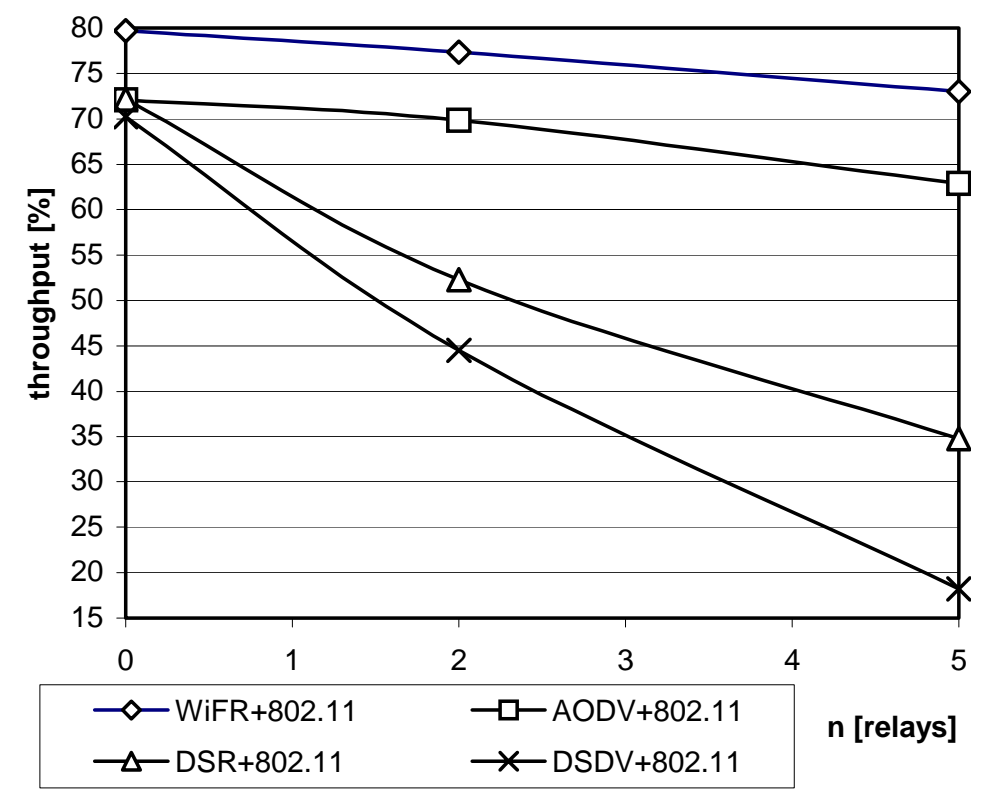

Fig. 5. WiFR vs ad-hoc routing protocols 


\section{Conclusion}

In this paper a new mathematical programming model has been proposed to describe QoS routing problem for infrastructure-based multi-hop wireless networks. The model is an extension of the well known multi-commodity flow problems where link capacity constraints are replaced with new ones that takes into account interference constraints among different radio links. Feasible solutions of the problem guarantee that the rates of routed flows are compatible with radio channel capacity, but does not require to explicitly solve the scheduling problem. We have also proposed a routing algorithm, Wireless Fixed Relay routing (WiFR), based on an adhoc heuristic to find feasible solutions in reasonable time. Numerical results, obtained with simulation, have show the effectiveness of WiFR to provide a network capacity much higher than the routing schemes proposed for ad-hoc networks and even higher than that of WSPF.

\section{Acknowledgements}

Part of this work has been performed in the framework of the IST project IST-2003507581 WINNER, partly funded by the European Union, and the FIRB project TANGO, partly founded by the Italian Ministry of Education and Scientific Research. The authors would like to acknowledge the contributions of their colleagues in WINNER and TANGO, although the views expressed are those of the authors and do not necessarily represent the projects.

\section{References}

1. https://www.ist-winner.org

2. C. Perkins, Ad Hoc on Demand Distance Vector (AODV) Routing, Internet draft, 1997.

3. D. B. Johnson and D. A. Maltz, Dynamic Source Routing (DSR) in Ad Hoc Wireless Networks, Internet draft, 1997.

4. C. Perkins, E. Royer, S. R. Das and M. K. Marina, Performance Comparison of Two OnDemand Routing Protocols for Ad Hoc Networks, IEEE Personal Communications, vol. 8, no. 1, Feb. 2001, pp. 16-28.

5. Z. J. Haas and M. R. Pearlman, "The Zone Routing Protocol (ZRP) for Ad Hoc Networks," Internet draft, 1997.

6. S. Chakrabarti and A. Mishra, QoS Issues in ad hoc wireless network, IEEE Communications Magazine, vol. 39, no. 2, Feb 2001, pp. 142-148.

7. E. Crawley et al., "A Framework for QoS-Based Routing in the Internet," RFC 2386, Aug. 1998.

8. J.L. Sobrinho and A.S. Krishnakumar, Quality-of-Service in ad hoc Carrier Sense Multiple Access Wireless Networks, IEEE JSAC, vol. 17, no. 8, Aug. 1999, pp. 1353-1414.

9. A. Iwata et al., Scalable Routing Strategies for Ad Hoc Wireless Networks, IEEE JSAC, vol. 17, no. 8, Aug. 1999, pp. 1369-79.

10.C.R. Lin and J.-S. Liu, QoS Routing in Ad Hoc Wireless Networks, IEEE JSAC, vol. 17, no. 8, Aug. 1999, pp. 1426-38. 
11.S. Chen and K. Nahrstedt, Distributed Quality-of-Service Routing in Ad Hoc Networks, IEEE JSAC, vol. 17, no. 8, Aug. 1999, pp. 1488-1505.

12.C.R. Lin, On-demand QoS routing in multihop mobile networks, IEEE INFOCOM 2001. April 2001, vol. 3, pp. 1735-1744.

13.Chenxi Zhu and M.S. Corson, QoS routing for mobile ad hoc networks, IEEE INFOCOM 2002, June 2002, vol. 2, pp. 958-967.

14.Hongxia Sun, H.D. Hughes, Adaptive QoS routing based on prediction of local performance in ad hoc networks, IEEE WCNC 2003, March 2003, vol. 2, pp. 1191- 1195.

15.M.Garey and D. Johnson. Computers and Intractability: a Guide to the Theory of NPCompleteness. W.H. Freeman, 1979.

16.H. Li, M. Lott, W. Zirwas, M. Weckler, E. Schulz, Multihop Communications in Future Mobile Radio Networks, IEEE PIMRC 2002, Sept. 2002.

17.H. Li, M. Lott, W. Zirwas, M. Weckler, E. Schulz, Hierarchical Cellular Multihop Networks, EPMCC 2003, March 2003.

18.A.A. Assad, "Multicommodity network flow - a survey". Networks, vol. 8 pagg. 37-91, John Wiley \& Sons, Inc.

19.K. Aardal, S.P.M. van Hoesel, A. Koster, C. Mannino, and A. Sassano, Models and Solution, Techniques for Frequency Assignment Problems, 4OR , (1) 4, 261-317, 2003.

20.http://www.isi.edu/nsnam/ns 